\title{
Early Detection Research Network in the US
}

\section{INTRODUCTION}

Over the past year, two review groups of outside experts (the Cancer Prevention Program Review Group and the Cancer Control Program Review Group) met to give advice to the National Cancer Institute (NCI). After the review groups had submitted their reports to the NCI and to the Board of Scientific Advisors, a number of implementation working groups were established. One of the working groups, the Early Detection Implementation Group (EDIG), was created to address the major recommendations made by the Cancer Prevention Program Review Group (CPPRG) in early cancer detection. Only relevant conclusions from the CPPRG report are presented here:

- develop new molecular markers for the early detection of cancer

- expand identification of high risk healthy populations based on genetic predisposition and the development of new molecular markers

- develop and improve new high through-put technologies for implementation of promising molecular diagnostic approaches in clinical and population-based trials

- develop and expand existing biorepositories and provide new access with appropriate consent to such materials for the testing of new molecular detection strategies.

The EDIG met four times to review all early detection-related recommendations made by the CPPRG in order to propose strategies for their implementation (the meeting dates and members of the EDIG are included in attachment). The EDIG endorsed the formation of a consortium to accelerate the progress made in the area of molecular and genetic markers toward application in cancer prevention, earlier detection and risk assessment. This concept represents one of the major recommendations made by the EDIG. The
Chemoprevention Implementation Group, the Breast Cancer Progress Review Group and the Prostate Cancer Progress Review Group independently made similar recommendations.

\section{BACKGROUND}

Although the primary tumor can usually be controlled by local therapy, most cancer deaths are caused by metastatic disease. The goal of early detection and screening is therefore the diagnosis and treatment of cancer before it spreads beyond the organ of origin, perhaps even in its pre-invasive state. Unfortunately, available early detection and screening techniques pick up many tumors at a relatively late stage in their natural history. As a result, decrements in mortality even with the best available detection modalities are likely to be modest. On the other hand, some early detection and screening techniques identify changes with a low probability of progression to life-threatening cancer, thereby resulting in unnecessary diagnosis and over-treatment. New technologies coming from the field of molecular and cellular biology are able to identify genetic as well as antigenic changes during the early stages of malignant progression. Some of these changes show promise as biomarkers for preneoplastic development or for early malignant transformation. The application of these emerging technologies in the field of early detection and risk assessment is a high priority in the National Cancer Institute's strategy for reducing mortality from cancer. Detection of early cancer has been identified as an area of extraordinary opportunity for investment in the NCI 1999 Bypass Budget.

Data show that detection and treatment of premalignant or early lesions can reduce mortality, for instance, mammography and Pap screening. Although clinically proven, both technologies have problems with sensitivity, specificity as well 
as predictive value. Therefore, it seems reasonable to explore the application of the new molecular-based technologies for earlier and more specific detection and even for risk assessment, that is, before the cancer physically develops in order to institute chemoprevention. These are the overarching goals of the proposed research network.

Early detection technologies are rapidly evolving while existing technologies are undergoing progressive refinement in their sensitivity, specificity, and throughput. Improved analytic tools have allowed more detailed examination of the molecular basis of carcinogenesis and provided the ability to identify the molecular and cellular signatures of cancer and explore geneenvironment interaction relevant to early detection. To fully explore the application of molecular profiles for earlier detection, it is essential to understand the molecular pathogenesis of cancer, that is, the natural history of tumor progression at the molecular level so that the biological behavior of an evolving lesion (for example: dysplasia or field change) can be predicted with greater accuracy. Current observations indicate that cancers usually evolve through many complex cellular processes, pathways, and networks. A better understanding of the circuits in these pathways is critical if we are to successfully apply these molecular-based technologies to earlier detection.

Research in molecular genetics, cell biology, protein chemistry and immunology has found that cells undergo many changes during neoplastic progression. Often occurring early in the malignant process, these changes include, for example, production of novel proteins, growth factors, cytokines, etc., in addition to multiple genetic alterations. Because these changes have been consistently associated with malignant transformation, they are now recognized as biomarkers for cancer, but are not always predictive. Such biomarkers, whether present in serum, urine, etc., could serve as indicators of early cancer or as markers of risk for impending cancer.

Progress in the field, however, is currently impeded by some practical hurdles. The systematic application of biomarkers for earlier cancer de- tection or even for risk assessment is fragmented and not well coordinated. For many years, the National Cancer Institute has sponsored research in genetic and molecular biomarkers through traditional funding rnechanisms, such as ROls and POls. While studies conducted through these mechanisms have been useful in advancing our understanding of carcinogenesis, there has been a lack of research emphasis on the continuum of preclinical tumor development, early evaluation of new techniques and their clinical application. In many of these reported studies the investigators did not fully explore the biological implications or systematically test the clinical application of these molecular markers. This has resulted, in part, from the lack of a stable connection between basic laboratory research and the opportunity for rapid clinical evaluation. Other factors contributing to the lack of systematic evaluation include the non-availability of high quality matched specimens from normal, suspicious, preneoplastic and multistage neoplastic lesions. In addition, the lack of large uniform collections of well-defined preneoplastic and neoplastic lesions, collected with ancillary demographic and follow-up clinical data, has also limited progress in the development and application of these biomarkers. As a consequence, much work in this area is fragmented into numerous small and disconnected studies without complete evaluation. Usually, the results of these studies cannot even be generalized to the population as a whole. In many instances, the population of inference cannot be defined.

\section{GOALS AND MAJOR OBJECTIVES}

The initiative will support the creation of a multi-center network with resources for translational research that will include the laboratory sciences, clinical sciences, public health, biostatistics, informatics, and computer sciences. The initial goals of the network will be to discover and to coordinate the evaluation of biomarkers/reagents for the earlier detection of epithelial cancers, such as prostate, breast, lung, colorectal and upper aerodigestive tract, and for the assess- 
ment of risk. Specifically, the objectives of the network will include:

- the development and testing of promising biomarkers or technologies in institutions having the scientific and clinical expertise, in order to obtain preliminary information that will guide further testing;

- the timely and early phase evaluation of promising, analytically proven biomarkers or technologies. Evaluation will include measures of diagnostic predictive value, sensitivity, specificity, and whenever possible, medical benefits, risk, and harms, such as predictors of clinical outcome or as surrogate endpoints for early detection and for prevention intervention clinical trials;

- the timely development of biomarkers and expression patterns, sometimes of multiple markers simultaneously, which will serve as background information for subsequent large definitive validation studies in the field of cancer detection and screening;

- collaboration among academic and industrial leaders in molecular biology, molecular genetics, clinical oncology, computer science, public health, etc., for the development of high throughput, sensitive assay methods for biomarkers from an early detection viewpoint;

- conducting early phases of clinical/epidemiological studies, e.g. cross-sectional, retrospective, to evaluate predictive value of biomarkers; and

- encouraging collaboration and rapid dissemination of information among awardees to ensure progress and avoid fragmentation of effort.

The ultimate impact of new technology on prolonging survival and reducing mortality will not be felt until highly predictive biomarkers are developed for earlier cancer detection or risk assessment.

The success of this effort depends in large measure on exploring the concordance between genetic or molecular markers and the morphologic changes associated with premalignant and pre-invasive lesions that have life-threatening potential. In other words, we need to identify biomarkers that are predictive of clinical outcomes. Surrogate endpoint biomarkers could provide biologic insights in the short-term, and eventually provide a rationale for changes in the design of clinical trials.

\section{ORGANIZATIONAL STRUCTURE}

\section{Definitions}

Network: This refers to a web of different intercommunicating components with defined functions and responsibilities in an overall organization. In this case, it refers to the overall organizational structure of the research proposal.

Consortium: This refers to a group of institutions conducting research in various scientific disciplines within the Network. In this proposal, it refers to the group of individual institutions in the network (i.e. the circle in the attached figure).

Network: The Network will consist of four components: 1) Consortium for Biomarkers in Early Detection Research (CBEDR), 2) a Steering Committee (SC), 3) an Advisory Committee (AC), and (4) a Data Management and Coordinating Center (DMCC) (Figure 1).

Consortium for Biomarkers in Early Detection Research: The CBEDR will consist of three main scientific components: i) Biomarker Developmental Laboratories (BDL), ii) Biomarker Validation Laboratories (BVL), and iii) Clinical and Epidemiologic Centers (CEC). Each laboratory/ center, which will be managed by a Principal Investigator, will include academic and industrial biotechnology investigators who are involved in cancer detection and diagnostic research. In order to expedite the translational research, the Consortium will be supplemented by the ad hoc participation of additional institutions (academic or community-based) that are able to validate the results of laboratory studies through patient accrual. The work of the Consortium will be coordinated by the Steering Committee (Figure 1).

It is anticipated that the CBEDR will consist of experts in basic molecular science, laboratory technology, clinical studies, biometry, and epidemiology. The expertise in laboratory science 


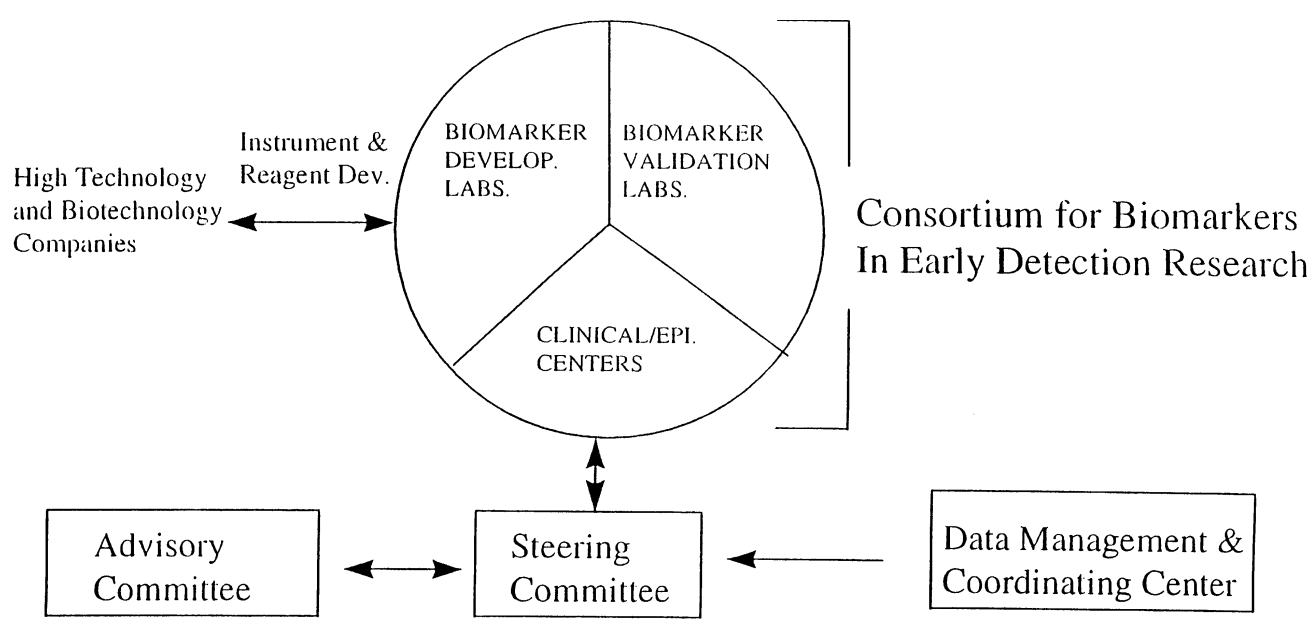

Fig. 1. Early detection research network.

should include conducting research in the basic biology of preneoplasia encompassing the development and testing of biomarkers of early cancer, development of relevant technologies for biomarker detection, and analytical tools for the evaluation of biomarkers for risk assessment. The expertise in laboratory validation should include knowledge and practice of Standard Operating Procedures (SOPs), and experience in statistical evaluation methodologies for checking accuracy, precision, reproducibility and performance characteristics of tests in multicenter settings. Expertise in patient accrual and associated clinical issues for pilot studies will be needed to apply basic science discoveries to clinical settings. Computational and informatic needs of the Consortium will be Supported by the Data Management and Coordinating Center. Experts in informatics will include database developers, combinatorial data analysts, and computer application program developers/specialists.

Research Member in the Consortium: Participation in the Consortium will require expertise in one or more of its scientific components. An applicant may seek funding to participate in more than one component. They will conduct research of the consortium using their core funds supplemented in some cases, as noted below.

Steering Committee: The SC will be composed of the Principal Investigators and Co-Principal Investigators from each member of the Consor- tium, the director of the Data Management and Coordinating Center, NCI program staff, and the chairperson of the Advisory Committee. The chair of the Steering Committee will be elected by the members of the Consortium. The Committee will decide on the frequency and content of meetings.

The Steering Committee will have major scientific management oversight, including monitoring the activities of the DMCC. Specifically, it will develop uniform criteria for the collection of clinical data, collection of tissue and blood specimens, and for instituting laboratory quality assurance. In addition, the Committee will develop common informatic and analytical tools for the interpretation of data and instruments for checking uniformity, consistency, accuracy and reproducibility of the data. It will study applied and theoretical approaches to the simultaneous analysis of multiple markers. The informatics support will be provided by the DMCC.

Advisory Committee: The AC will include members who are not participating in the Consortium. Each Principal Investigator in the consortium will be asked to nominate members for the AC. The membership to the Committee and duration will be decided by the NCI in consultation with the Steering Committee. The AC will include basic scientists, clinicians, prevention scientists, epidemiologists, ethicists, statisticians, and members from relevant advocacy groups. 
Scientific experts will be drawn from various disciplines relevant to multi-center detection research and experts in data management, biostatistics, and clinical study design. The Chair of the AC will be elected by its members. The Chairperson of the Steering Committee will also serve as a member of the Advisory Committee. The NCI will be represented by the relevant program staff.

The AC will independently advise the Steering Committee on relevant scientific issues, including study design, prioritization of biomarker development, development of study protocols, including decision criteria for clinical applications, e.g., early detection, prognosis, etc. The AC will also advise the Steering Committee when to move discoveries from the laboratory into clinical testing using appropriate patient groups, and evaluate the progress and success of the Network. Prospective evaluation criteria for the success of the Network will be developed.

\section{Data Management and Coordinating Center}

The DMCC will provide logistic support for the conduct of the Steering and Advisory Committee meetings, provide statistical and data management support for protocol development, conduct analysis, and informatics. It will study applied and theoretical approaches to the simultaneous analysis of multiple markers.

Funds: Operating funds will reside with 1) the Consortium For Biomarkers in Early Detection Research, 2) the Data Management and Coordinating Center, and 3) the Steering Committee.

\section{Consortium for Biomarkers in Early Detection Research}

The Principal Investigators will have funds available to support the development of the scientific program and clinical protocols. Collaborations will also be extended to investigators who are engaged in translational research on biomarkers, but are not funded through the consortium. It is expected that the Steering Committee will establish guidelines for including such investiga- tors. Core funds will be made available for such investigators in order to include valuable clinical collaborators who may come from outside the core group as well as from industry. All investigators will be encouraged to seek supplemental funding through SBIR/STTR, R21/R33, and other research mechanisms.

\section{Data Management and Coordinating Center}

The DMCC will be funded through a separate RFA. While the NCI plans to proceed with the funding request, its publication and timing will depend on the establishment of the Consortium and the Steering Committee first.

\section{Core Funds for the Steering Committee}

Discretionary funds will be available to the Steering Committee. Core funds can be used for a variety of functions:

1. Core funds would be used to expand the membership of the consortium through supplemental funding to an investigator's current funded grants.

2. Funds will often be needed in moving a new marker test to the point at which it can be validated at multiple centers and in larger populations. Test reagents will require scale-up at this point, and the Steering Committee will require sufficient funding to contract to laboratories or companies that can scale up production and maintain quality of the reagents (e.g. monoclonal antibodies, labels, etc.). Funds will also be required for data management, travel, group meetings, and other core activities of the group.

INTERACTION WITH THE CLINICAL TRIAL/TREATMENT COMMUNITY: Plans will include collaboration with other NIH Institutes and government agencies or departments (e.g., FDA, DOD, VA), with other NCI programs (e.g., SPOREs, Cancer Genetics Network, Breast and Colon Cancer Family Registries, Cooperative Human Tissue Network), with ongoing NCI clinical research programs (e.g., CCOP, PLCO), and with active research groups with ongoing trial core functions and laboratory support such 
as the Cooperative Groups, NCI designated cancer centers, international collaborators, clinical epidemiologists, and health maintenance organizations interested in early detection research. Collaboration with the DOD and VA may be expedited by the Agreement that NCI has signed, which calls for joint research in cancer prevention and early detection.

INTERACTION WITH INDUSTRY: A major difficulty facing industry is the lack of access to clinical environments for technology assessment. Creation of the Network will serve as an attractive collaborator for industry, since it will provide clinical opportunities for the evaluation of new technologies. The Network will encourage collaboration with industry on a substantial costsharing basis. NCI funds will be used to support the underlying infrastructure and the cost of studies not having direct implications for a company's product development or marketing strategy. However, for new technologies that are part of a company's development or product plans, the individual companies will be responsible for costs in such areas as technology standardization and quality assurance as well as scale-up of laboratory techniques, in collection and formatting of specialized data required by regulatory agencies for device approvals, in the preparation of registration documents, and in supporting a portion of the accrual to studies pivotal for registration. It is anticipated that industry participating in the Network will not charge investigators or NCI for technologies/reagents that will be evaluated in collaborative studies. NCI views the partnership with industry as an important component without resorting to the subsidization of private companies.

Governance: The Principal Investigator will be responsible for administering and supervising research personnel, and for conducting research. The Principal Investigator will also be responsible for the expenditure of the annual budgets.

The Steering Committee will be responsible for coordinating the research effort across the Consortium, including the Data Management and Coordinating Center, and may formulate direc- tives that will govern the operations of the Consortium. A simplified example is provided that illustrates the functions of the Consortium and the support it offers for moving basic research findings into clinical practice.

An investigator within the Consortium identifies a putative biomarker through original laboratory research. Based on the pilot research findings, the putative marker seems to be useful for early cancer detection. The investigator can then approach the Steering Committee for additional evaluation of the marker and possible support for further testing. The Steering Committee then has the responsibility to review the data on the potential marker using its standing formal criteria as a guide. The Steering Committee can consult the Advisory Committee to obtain information on the requirements and need for additional research on the marker. It also can consult the Biomarker Validation Laboratories and the Clinical Centers regarding requirements for laboratory tests, needs for quality assurance, and the availability of patient groups for clinical validation. If necessary, resources from other Centers can be pooled to conduct studies. Concurrently, the informatic team in Data Management can develop tools for the analysis of results.

There will also be flexibility so that investigators outside the Consortium could form a collaboration with one of the existing centers, or directly bring their discoveries to the Steering Committee (e.g., By Letter of Intent). To support such efforts, the Steering Committee will be able to use core funds to supplement the investigator's ongoing research. The investigator, in turn, will agree to share his research findings and become part of the Consortium.

\section{JUSTIFICATION FOR COOPERATIVE AGREEMENT}

The mechanism of support will be the Cooperative Agreement. This mechanism is appropriate because the participating organizations will have the responsibility for defining the scientific 
objectives and approaches needed. The purpose of this RFA is to encourage and to facilitate interdisciplinary collaboration among organizations (institutes and/or consortia) toward a common coordinated national effort in research in early cancer detection. Substantial NCI involvement is anticipated in order to: 1) facilitate interaction among the centers, 2) coordinate their efforts with other ongoing NCI and non-NCI initiatives, 3 ) promote the use of this resource among the scientific and medical communities, and 4) solicit the presentation of research proposals requesting the utilization of the laboratory science discoveries, epidemiologic data and biologic specimens. It is anticipated that prioritization of proposals requesting access to technologies/reagents developed through the Consortium for Biomarkers in Early Detection Research will be made by the Steering Committee in consultation with the Advisory Committee.

\section{CURRENT PORTFOLIO ANALYSIS}

There are no standing activities supported by NCI that have as the goal the "vertical", sequential development of biomarkers for early detection, beginning with basic discovery, to translational research, and to clinical validation. Also, there is no existing NIH mechanism to expedite technology transfer across a broad spectrum of sciences applicable to technology development from laboratory to clinical application. Portfolio analysis suggests that developmental studies in biomarker research that originate in academic centers have nowhere to go for definitive comparative testing or for validation, because there is little formal connection between biomarker laboratory research and clinical application. Microsatellite analysis, for example, has been rigorously tested for its analytical accuracy and found to be associated with the early phases of tumor development. However, its value in screening is yet to be determined. Hereditary cancer markers, such as APC, hMSH2, HMLH1, hMLH2, and other serum-based markers, such as PSA, and
CA-125, are being widely used without having undergone validation and knowing if there is net benefit or harm as a result of their application. A gap exists between basic research in preclinical early detection and the means for clinical testing. At present, investigators develop molecular and genetic markers, and then move on to new markers without validating the utility of previous ones. Validation studies do not often fare well within the present peer-review system, because these studies are not necessarily considered innovative, and they are also considered expensive.

\section{PROGRAM EXPECTATION/ EVALUATION}

The establishment of improved strategies for the identification of individuals with small neoplastic or preneoplastic lesions with reasonable probability of progression (and that are amenable to cure) is the primary goal of this research program. It is anticipated that the research will develop and evaluate an ensemble of biological markers that will indicate the presence of early cancer or preneoplastic events. An ensemble of markers is likely to be more useful and a better predictor of disease status than a single marker or a narrow range of markers that might focus only on one or two pathways in carcinogenesis. Such a strategy will require a multidisciplinary, multiinstitutional approach, such as the Network presented here.

There is no precedent for this model. However, the present concept has been inspired by the successful launching of The Cooperative Trials in Diagnostic Imaging by the Diagnostic Imaging Program (DIP). The Early Detection Research Network will closely interact with DIP and learn from its experience to periodically evaluate the success of this model. It will involve the members of the various advisory groups, such as the Board of Scientific Advisors and the National Cancer Advisory Board, to help evaluate the program against the criteria established by the Advisory Committee. 


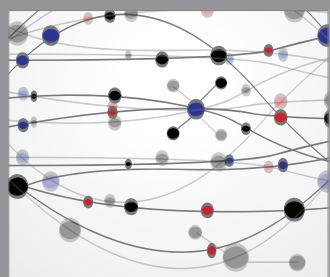

The Scientific World Journal
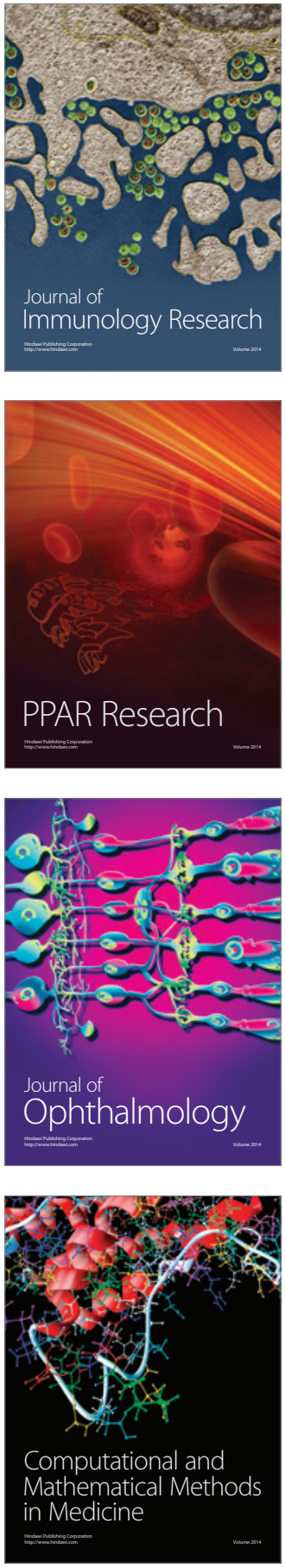

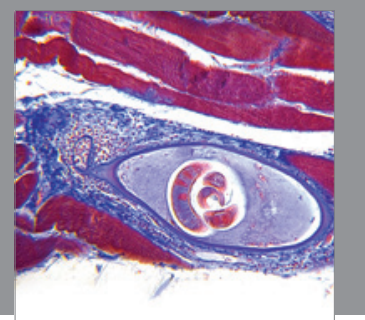

Gastroenterology

Research and Practice
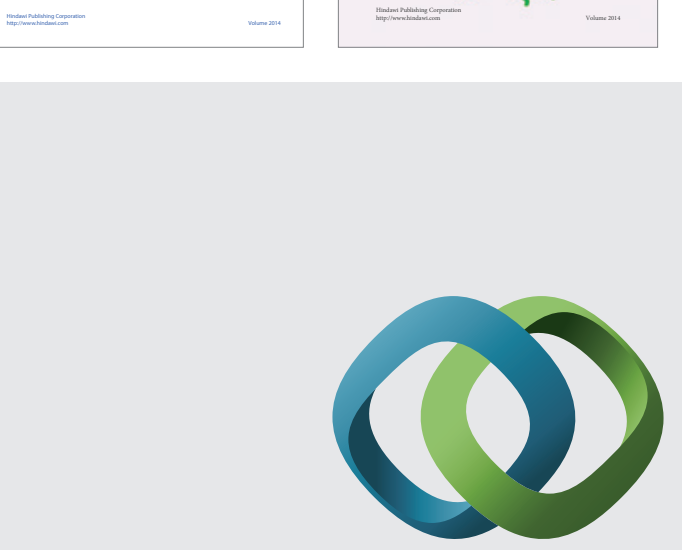

\section{Hindawi}

Submit your manuscripts at

http://www.hindawi.com
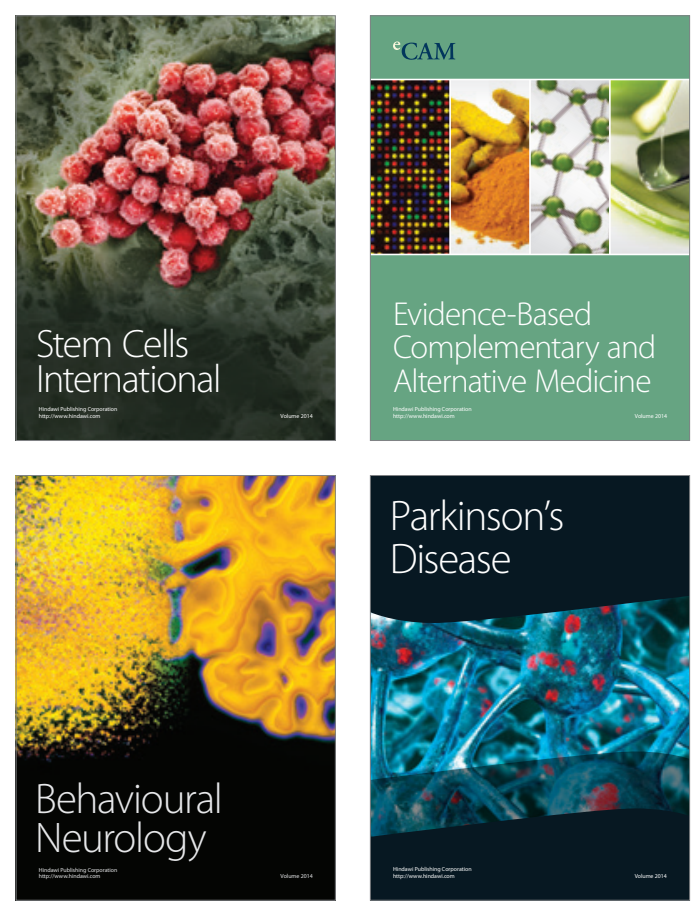

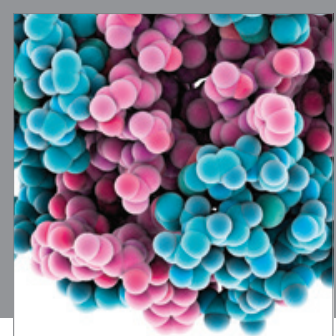

Journal of
Diabetes Research

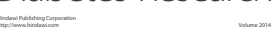

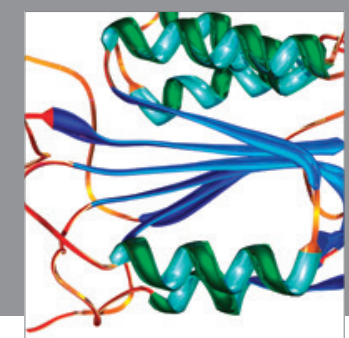

Disease Markers
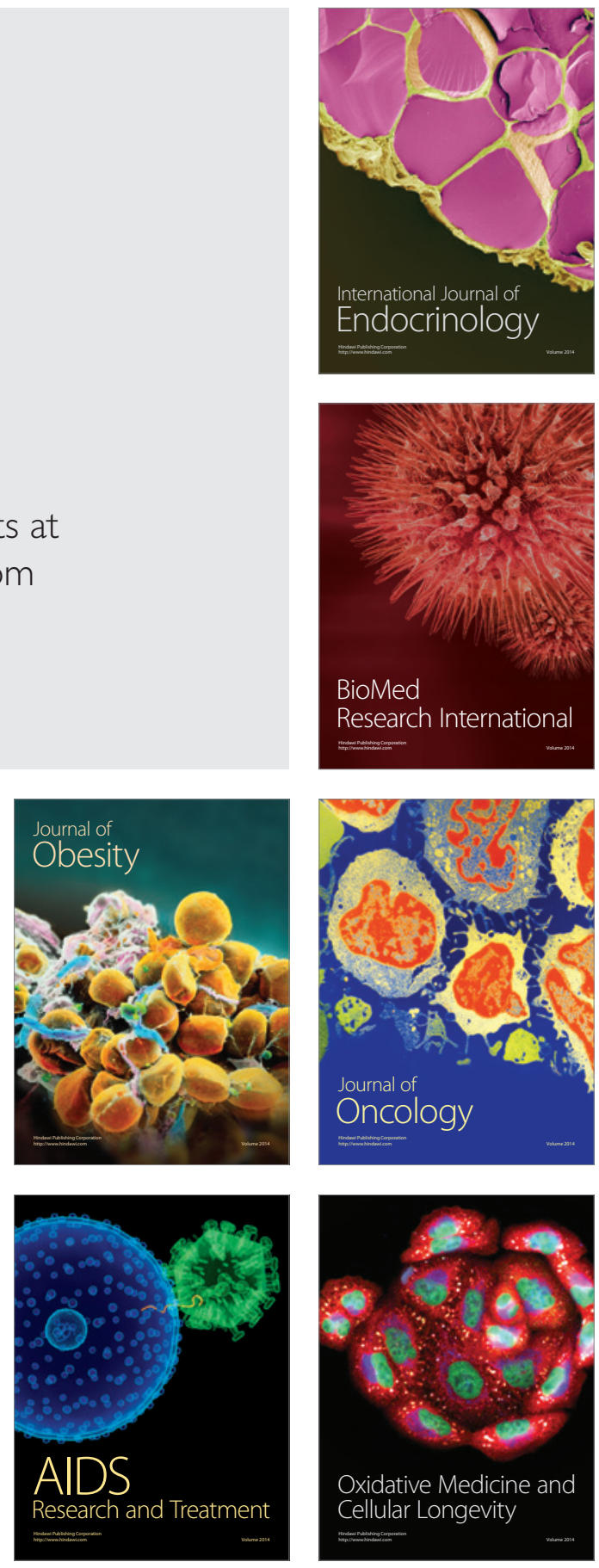